\title{
UAV adaptive trajectory tracking control under unknown time-varying disturbance and input saturation
}

\author{
Yimin Zhou ${ }^{\mathrm{a}, *}$, Zengwu Tian ${ }^{\mathrm{a}, \mathrm{b}}$ \\ ${ }^{a}$ Shenzhen Institute of Advanced Technology, Chinese Academy of Sciences, Shenzhen, \\ China. \\ ${ }^{b}$ University of Chinese Academy of Sciences, Beijing, China.
}

\begin{abstract}
In this paper, the flight control of the Unmanned aerial vehicle (UAV) is discussed with the proposed adaptive dynamic surface control method owing to its underactuated and non-linear characteristics. The proposed control algorith$m$ is based on radial basis function (RBF) neural network and anti-saturation auxiliary system to realize high-precision trajectory tracking under time-varying disturbances and input saturation. First, the nonlinear dynamic model of the UAV with disturbances is established with the aid of rigid body motion theory. With the adoption of the dynamic surface control algorithm, the error surface and the Lyapunov function are defined to design the preliminary control law of the designed controller. Then the RBF neural network is introduced to estimate and compensate the disturbance. Further, an anti - saturation module is designed to tackle the problem of input saturation. By using the Lyapunov stability theory, it is proved that the stability and signal consistency of the closed-loop system are bounded, along with the constrained conditions of the control parameters. Simulation experiments have been performed and the results demonstrate that the proposed control algorithm has high-precision trajectory tracking ability and strong anti-disturbance capability under the input
\end{abstract}

\footnotetext{
* Corresponding author

Email address: ym.zhou@siat.ac.cn (Zengwu Tian)
}

Preprint submitted to Nonlinear Dynamics

August 14, 2021 
saturation constraint with high control performance.

Keywords: Adaptive control, Input saturation, Unknown time-varying

disturbance, Trajectory tracking control, UAV

\section{Introduction}

The Unmanned Aerial Vehicle (UAV) has the characteristics of simple structure, light weight, easy to carry and strong reliability which has been widely used in diversified applicable fields, becoming a research hotspot [1-2]. However, owing to the high nonlinearity and strong coupling of the quadrotor system, the controller design of the UAV is highly challenging [3]- 4 .

The linear control methods are rather easy to be analyzed and simple to be designed, thus they have been widely and generally applied to UAVs. In [5], two kinds of control systems based on PID algorithm are designed, which can achieve the control stabilization of the quadrotor. A multi-Objective high exploration particle swarm optimization is used for linear quadratic regulator (LQR) optimization for a 4 Degree-Of-Freedom (DOF) quadrotor [6], while the experiment results demonstrate the control capability of UAV to reach and track a given trajectory. An adaptive model predictive controller based on Laguerre is designed for the attitude stabilization of the quadrotor in a real-time low-power embedded platform [7]. Besides, a robust mixed $H_{2} / H_{\infty}$ tracking controller is designed with static state feedback for attitude tracking of a UAV. Further, with the added regional pole-placement constraints, the response of the mixed $H_{2} / H_{\infty}$ controller can be improved greatly [8]. As a popular robust control method, the linear ADRC (Active Disturbance Rejection Control) approach has been utilized for the quadrotor control, achieving the desired attitude and height successfully [9]. As it is known that most linear controllers are simple in design, however, they can only guarantee the stability of UAV at the equilibrium point. Moreover, some linear controllers has limited dynamic performance that 
high control accuracy cannot be obtained. Therefore, it is necessary to further study the nonlinear control of UAVs.

Recently, more and more researchers have applied nonlinear control on UAVs. In [10, a nonlinear PID controller is developed with an adaptive neuronal technique, where the output errors are re-propagated to adjust the PID gains to reduce the control errors. Simulation results identify that this method has a higher control performance than the traditional PID method. In [11, the backstepping approach is applied for the control of the aircraft attitude and translational displacement, while sliding mode control algorithm is used for altitude control. The proposed controller has been tested in an outdoor environment with high control reliability. A fuzzy sliding mode based controller is designed for attitude control to stabilize the quadrotor, while the output from the fuzzy sliding mode controller can be utilized to estimate the switching function, thus achieving fast response speed and high robustness [12. Further, a model-freebased terminal sliding mode controller has been developed to control the attitude and position of a quadrotor with higher dynamic performance [13]. A dual BP networks are applied in an adaptive inverse model control system for UAV control, where the BP network are trained offline preliminary and online update as well to guarantee the quality of the model identification so as to maintain the UAV flight stability [14]. A neuroadaptive dynamic surface containment control system [15] is designed based on minimal learning parameter of the estimator for the transient and steady-state flight control of multiple quadrotors under uncertainties.

It is aware that UAV is easily affected by external disturbances during the flight, hence, it is necessary to study the flight control of UAV under disturbances [16]. In [17, a disturbance observer based on Simpson's approximation is developed and incorporated with sliding mode control which can greatly mit- 
igate the impact of the input saturation. In [18, a path-following controller based on an uncertainty and disturbance estimator (UDE) is proposed for a quadrotor with a cable-suspended payload, which can stabilize the quadrotor with the desired path under different wind disturbances. A novel nonlinear control approach with two observer-based estimators [19] is proposed to precisely estimate the disturbance torque, where the disturbance can totally be compensated. Further, a disturbance detection method with the IMU sensor is proposed for attitude recovery control with faster response to the disturbance [20]. Moreover, a nonlinear discrete-time disturbance observer is proposed based on the backstepping strategy with the approximation of the neural network to constrain the disturbances in boundary [21]. In [22], a global controller with nonlinear adaptive state feedback is designed for the quadrotor trajectory tracking under constant wind disturbances. Besides, online adaptive rules are established for the quadrotor control to estimate the upper bound of the uncertainties and disturbances of the attitude in the presence of parametric uncertainties [23].

It is known that if more torques are required from the controller than that provided by the actuators, the system control performance would be degraded or result in mechanical failure. Thus the control input saturation is an important factor which could affect the flight stability of the UAV 24]. To stabilize UAV flight along with the desired status with input saturation, a PD (proportionalderivative) control algorithm is proposed on special Orthogonal-3 to quickly converge to the desired attitude 25. A suboptimal integral sliding mode controller (ISMC) is designed where the state dependent Riccati equation can be used for online feedback of the input saturation [26]. Further, a backstepping based adaptive fault tolerant control method [27] is proposed, where the input saturation can be dealt with a command filter and simulation experiments confirm that the actuator faults are bounded. In [28, a body-rate controller with 
motor saturation is presented for UAV stable trajectory tracking.

A UAV adaptive controller is proposed for trajectory tracking under timevarying and asymmetric output constraints, and the upper bound of the external interference can be estimated online via the asymmetric Barrier Lyapunov Function 29. The commonly used PD plus dynamics compensation scheme can be extended where the linear errors are replaced by the saturated non-smooth but continuous exponential-like ones, so as to reduce the actuator failure owing to excessive input torque [30.

UAVs are easily affected by the disturbances which are difficult for UAV to ensure high-precision trajectory tracking without anti-disturbance design. Disturbance observer is normally used for the disturbance rejection but with the physical model requirement. In this paper, we consider to achieve disturbance estimation so as to reduce the dependence on physical model information. In order to realize high precision control of the UAV, RBF neural network without model information is introduced for disturbance estimation based on dynamic surface nonlinear control. Hence, an unknown time-varying disturbance and input saturation are considered in the controller design to improve the trajectory tracking performance of the UAV. The contributions of the paper are summarized as follows:

1) The RBF neural network without model information is introduced in the control design, which can not only reduce the difficulty of disturbance rejection design, but also achieve disturbance estimate. Furthermore, the stability analysis is conducted to prove the effectiveness of RBF neural network theoretically.

2) An anti-saturation strategy is designed to avoid input saturation and proved via Lyapunov function with theoretical analysis and experimental verification.

3) The dynamic surface control algorithm, the RBF neural network and anti- 
saturation strategy are combined to design the composite controller to realize high-precision control of the UAV in complex environments. The range of the involved control parameters are provided during the stability analysis of the proposed composite controller.

4) A series of experiments have been performed to verify the UAV trajectory tracking behaviour with the proposed controller under the disturbance and input saturation constraint.

The remainder of the paper is organized as follows. In Section II, the UAV dynamic model with disturbance is established. In Section III, the controller design is described in detail and the stability of the control system is testified via Lyapunov stability theory. In Section IV, a series of simulation experiments are performed to verify the stability and efficacy of the proposed control method. Conclusion is provided in Section V.

\section{UAV dynamic modeling}

As it is known, UAV is a non-linear system and its horizontal motion is highly coupled with pitch and roll motions. To reduce the difficulty of the controller design, it is necessary and reasonable to simplify the UAV physical model as a rigid structure composed of four arms with the shape and mass symmetrical about the center and unchanged mass. The earth coordinate system and the body coordinate system are established to develop the UAV dynamics model, while the earth coordinate $\operatorname{system}\left(O_{e}, X_{e}, Y_{e}, Z_{e}\right)$ is with a fixed point of the earth as the origin and the body coordinate system $\left(O_{b}, X_{b}, Y_{b}, Z_{b}\right)$ is with the geometric center of the body as the origin, which is demonstrated in Fig. 1.

Suppose that the body coordinate system and the earth coordinate system coincide at the origin point, the transformation matrix from the body coordinate 


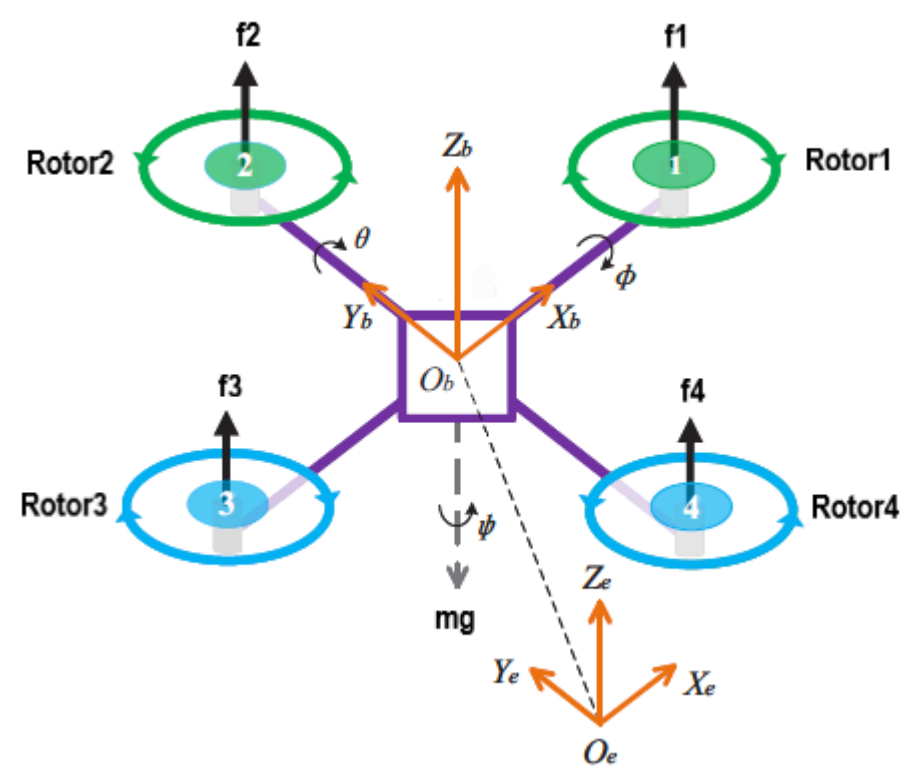

Figure 1: Schematic framework of a quadrotor UAV

system to the earth coordinate system is,

$$
\begin{aligned}
& R_{g / b}=\left[\begin{array}{cc}
\cos \psi \cos \theta & -\sin \psi \cos \varphi+\cos \psi \sin \theta \sin \varphi \\
\sin \psi \cos \theta & \cos \psi \cos \varphi+\sin \psi \sin \theta \sin \varphi \\
-\sin \theta & \cos \theta \sin \varphi \\
\sin \psi \sin \varphi+\cos \psi \sin \theta \cos \varphi \\
-\cos \psi \sin \varphi+\sin \psi \sin \theta \cos \varphi
\end{array}\right] \\
& \cos \theta \cos \varphi
\end{aligned}
$$

where $\{\psi, \theta, \varphi\}$ are the yaw angle, pitch angle and roll angle, respectively. In the earth coordinate system, the state-space equation of the quadrotor system 
is described as,

$$
\left[\begin{array}{c}
\ddot{x} \\
\ddot{y} \\
\ddot{z}
\end{array}\right]=R_{g / b} f \sum_{i=1}^{4} \Omega_{i}^{2}\left[\begin{array}{c}
0 \\
0 \\
1
\end{array}\right]+\left[\begin{array}{c}
0 \\
0 \\
-m g
\end{array}\right]+\left[\begin{array}{c}
\Delta_{4} \\
\Delta_{5} \\
\Delta_{6}
\end{array}\right]
$$

where $(x, y, z)$ is the position of UAV in the earth coordinate system, $f$ is the lift coefficient, $\Omega_{i}$ is the $i^{\text {th }}(i=1,2,3,4)$ rotor speed, $\left(\Delta_{4}, \Delta_{5}, \Delta_{6}\right)$ are the disturbances, $m$ is the mass of the UAV and $g$ is the acceleration of the gravity.

In the body coordinate system, the system state can be expressed as,

$$
\left\{\begin{array}{c}
\ddot{\varphi}=\left[l f\left(\Omega_{4}^{2}-\Omega_{2}^{2}\right)+\left(I_{x}-I_{z}\right) \dot{\theta} \dot{\psi}\right] / I_{x}+\Delta_{1} \\
\ddot{\theta}=\left[l f\left(\Omega_{3}^{2}-\Omega_{1}^{2}\right)+\left(I_{z}-I_{x}\right) \dot{\varphi} \dot{\psi}\right] / I_{y}+\Delta_{2} \\
\ddot{\psi}=\left[d\left(\Omega_{1}^{2}-\Omega_{2}^{2}+\Omega_{3}^{2}-\Omega_{4}^{2}\right)+\left(I_{x}-I_{y}\right) \dot{\theta} \dot{\varphi}\right] / I_{z}+\Delta_{3}
\end{array}\right.
$$

where $l$ is the distance between the rotor and the geometric center of the UAV, $d$ is the reverse torque coefficient of the motor, and $\left(I_{x}, I_{y}, I_{z}\right)$ are the moment of inertia of rotation about the axis $(x, y, z)$ respectively, $\left(\Delta_{1}, \Delta_{2}, \Delta_{3}\right)$ are the disturbances.

Here, it defines,

$$
\left[\begin{array}{c}
U_{1} \\
U_{2} \\
U_{3} \\
U_{4}
\end{array}\right]=\left[\begin{array}{c}
f \sum_{i=1}^{4} \Omega_{i}^{2} \\
l f\left(\Omega_{4}^{2}-\Omega_{2}^{2}\right) \\
l f\left(\Omega_{3}^{2}-\Omega_{1}^{2}\right) \\
d\left(\Omega_{1}^{2}-\Omega_{2}^{2}+\Omega_{3}^{2}-\Omega_{4}^{2}\right)
\end{array}\right]
$$

where $U_{1}$ and $U_{i}(i=2,3,4)$ represent the thrust and torque of the UAV, respectively. 
Finally, the nonlinear dynamic model of the UAV can be obtained as,

$$
\left\{\begin{array}{c}
\ddot{\varphi}=\left[U_{2}+\left(I_{x}-I_{z}\right) \dot{\theta} \dot{\psi}\right] / I_{x}+\Delta_{1} \\
\ddot{\theta}=\left[U_{3}+\left(I_{z}-I_{x}\right) \dot{\varphi} \dot{\psi}\right] / I_{y}+\Delta_{2} \\
\ddot{\psi}=\left[U_{4}+\left(I_{x}-I_{y}\right) \dot{\theta} \dot{\varphi}\right] / I_{z}+\Delta_{3} \\
\ddot{x}=(\sin \psi \sin \varphi+\cos \psi \sin \theta \cos \varphi) U_{1} / m+\Delta_{4} \\
\ddot{y}=(-\cos \psi \sin \varphi+\sin \psi \sin \theta \cos \varphi) U_{1} / m+\Delta_{5} \\
\ddot{z}=\cos \theta \cos \varphi U_{1} / m-g+\Delta_{6}
\end{array}\right.
$$

As it can be seen from Eq. (5), UAV is a nonlinear underactuated system with four inputs $\left(U_{1}, U_{2}, U_{3}, U_{4}\right)$ and six outputs $(\varphi, \theta, \psi, x, y, z)$. The detailed explanation of the UAV modelling is omitted here, and the interested readers can refer [11. Compared with the full actuated system, the controller design and analysis of the UAV are much more difficult while the designed controller for underactuated UAV is thoroughly explained in the next Section.

\section{Controller design and stability analysis}

In order to reduce the difficulty of the control design and guarantee the flight stability of the UAV, the UAV controller is designed as a cascade structure where the outer loop is the position control and the inner loop is the attitude control so as to achieve decoupling. The desired reference trajectory is set as $\left(x_{d}, y_{d}, z_{d}, \psi_{d}\right)$ and four independent control variables $\left(U_{1}, U_{2}, U_{3}, U_{4}\right)$ are used as the control inputs for the UAV. Thus the overall control block diagram is demonstrated in Fig. 2 .

According to Fig. 2, the desired roll angle $\varphi_{d}$ and the desired pitch angle $\theta_{d}$ are input in the attitude loop control. Since UAV is an underactuated system, 


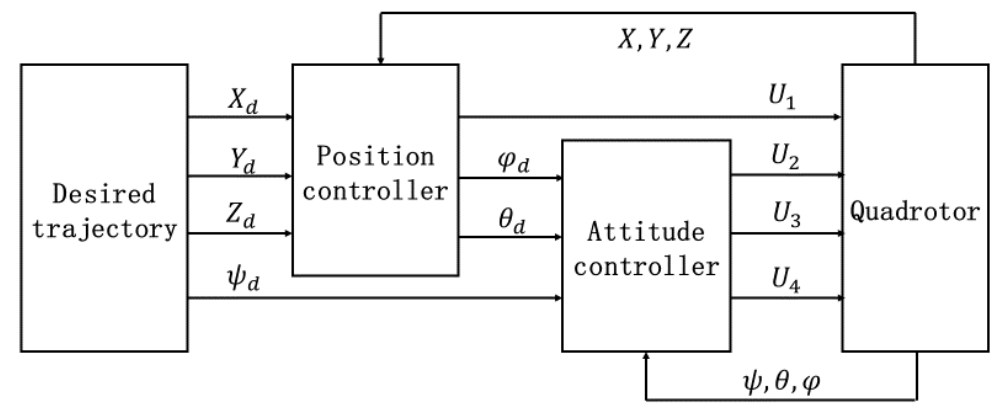

Figure 2: The control block diagram of the UAV

the intermediate variables $U x$ and $U y$ are introduced to calculate $\varphi_{d}$ and $\theta_{d}$,

$$
\left\{\begin{array}{c}
U_{x}=\sin \psi \sin \varphi+\cos \psi \sin \theta \cos \varphi \\
U_{y}=-\cos \psi \sin \varphi+\sin \psi \sin \theta \cos \varphi
\end{array}\right.
$$

Then $\varphi_{d}$ and $\theta_{d}$ can be calculated as,

$$
\left\{\begin{array}{l}
\varphi_{d}=\arcsin \left(U_{x} \sin \psi-U_{y} \cos \psi\right) \\
\theta_{d}=\arcsin \frac{U_{x} \cos \psi+U_{y} \sin \psi}{\cos \varphi_{d}}
\end{array}\right.
$$

According to the requirement of the cascade control, six controllers should be designed simultaneously. In this paper, each controller is designed individually, for the design convenience, the following variables are then defined as,

$$
\left\{\begin{array}{l}
\varphi=x_{11}, \dot{\varphi}=x_{12}, \theta=x_{21}, \dot{\theta}=x_{22}, \psi=x_{31} \\
\dot{\psi}=x_{32}, x=x_{41}, \dot{x}=x_{42}, y=x_{51}, \dot{y}=x_{52} \\
z=x_{61}, \dot{z}=x_{62}, \varphi_{d}=x_{1 d}, \theta_{d}=x_{2 d}, \psi_{d}=x_{3 d} \\
x_{d}=x_{4 d}, y_{d}=x_{5 d}, z_{d}=x_{6 d}
\end{array}\right.
$$


Thus Eq.(5) can be rewritten as the state equations,

$$
\left\{\begin{array}{c}
\dot{x}_{i 1}=x_{i 2} \\
\dot{x}_{i 2}=f_{i}(x)+b_{i} u_{i}+\Delta_{i}
\end{array} \quad i=1,2,3,4,5,6\right.
$$

where,

$$
\left[\begin{array}{c}
f_{1}(x) \\
f_{2}(x) \\
f_{3}(x) \\
f_{4}(x) \\
f_{5}(x) \\
f_{6}(x)
\end{array}\right]=\left[\begin{array}{c}
\left(I_{x}-I_{z}\right) \dot{\theta} \dot{\psi} / I_{x} \\
\left(I_{z}-I_{x}\right) \dot{\varphi} \dot{\psi} / I_{y} \\
\left(I_{x}-I_{y}\right) \dot{\theta} \dot{\varphi} / I_{z} \\
0 \\
b_{1} \\
b_{2} \\
b_{3} \\
b_{4} \\
b_{5} \\
b_{6}
\end{array}\right],\left[\begin{array}{c}
u_{1} \\
u_{2} \\
u_{3} \\
u_{4} \\
u_{5} \\
u_{6}
\end{array}\right]=\left[\begin{array}{c}
U_{2} \\
U_{3} \\
U_{4} \\
U_{x} \\
U_{y} \\
U_{1}
\end{array}\right]
$$

It is known that the cascade control is designed to realize the decoupling of UAV position and attitude, so that the dynamic equations can be simplified to the form of state equations for the convenience of subsequent controller design.

\subsection{Controller design}

The backstepping design control method is a recursive design method, where the main idea is to decompose the $n$ order control system into $n$ subsystems. The Lyapunov function is selected to design the control law of the subsystems respectively. In the backstepping control design, there exists a phenomenon of 
"differential explosion", which could cause the controller output to be too large [31]. Hence, a first-order low-pass filter is introduced to obtain the derivative of the virtual control of the backstepping method, thus forming a dynamic surface control design 32. Disturbance and input saturation limitation are further considered based on dynamic surface control method. The control design is as follows.

Based on Eq. $(9)$, the $1^{\text {st }}$ error surface is defined as,

$$
z_{i 1}=x_{i 1}-x_{i d}
$$

where $x_{i d}$ is the desired trajectory. Then the derivative of Eq.(11) is calculated as,

$$
\dot{z}_{i 1}=\dot{x}_{i 1}-\dot{x}_{i d}=x_{i 2}-\dot{x}_{i d}
$$

Hence, the Lyapunov function is defined as,

$$
\hat{V}_{i 1}=\frac{1}{2} z_{i 1}^{2}
$$

While the derivative of $\hat{V}_{i 1}$ is calculated as,

$$
\dot{\hat{V}}_{i 1}=z_{i 1} \dot{z}_{i 1}=z_{i 1}\left(x_{i 2}-\dot{x}_{i d}\right)
$$

According to Eq. (14), the virtual control can be selected as,

$$
\bar{x}_{i 2}=-k_{i 1} z_{i 1}+\dot{x}_{i d}
$$

where $k_{i 1}$ is the control coefficient.

In order to prevent the "differential explosion" caused by repeated integra- 
tion of $\bar{x}_{i 2}$, let $\bar{x}_{i 2}$ pass through a first-order low-pass filter, satisfying,

$$
\left\{\begin{array}{c}
\tau_{i} \dot{a}_{i}+a_{i}=\bar{x}_{i 2} \\
a_{i}(0)=\bar{x}_{i 2}(0)
\end{array}\right.
$$

where $\tau_{i}$ is the coefficient of the low-pass filter, and $a_{i}$ is the output of the filter.

Next, the $2^{\text {nd }}$ error surface is defined as,

$$
z_{i 2}=x_{i 2}-a_{i}
$$

The related derivative of Eq. 17] is calculated as,

$$
\dot{z}_{i 2}=\dot{x}_{i 2}-\dot{a}_{i}=f(x)_{i}+b_{i} u_{i}+\Delta_{i}-\dot{a}_{i}
$$

The related Lyapunov function is defined as,

$$
V_{i 1}=\frac{1}{2} z_{i 1}^{2}+\frac{1}{2} z_{i 2}^{2}
$$

The derivative of Eq. 19 is calculated as,

$$
\begin{aligned}
\dot{V}_{i 1} & =z_{i 1} \dot{z}_{i 1}+z_{i 2} \dot{z}_{i 2} \\
& =z_{i 1}\left(z_{i 2}+a_{i}-\dot{x}_{i d}\right)+z_{i 2}\left(f(x)_{i}+b_{i} u_{i}+\Delta_{i}-\dot{a}_{i}\right)
\end{aligned}
$$

According to Eq. 20, the preliminary control law is designed as,

$$
u_{i}=\frac{1}{b_{i}}\left(-f(x)_{i}+\dot{a}_{i}-\Delta_{i}-k_{i 2} z_{i 2}-z_{i 1}\right)
$$

where $k_{i 2}$ is the control coefficient.

In Eq. (21), $\Delta_{i}$ is the unknown disturbance and assumed as a continuous function. Considering that RBF neural network (RBFNN) can effectively ap- 
proximate arbitrary nonlinear continuous function [33], the RBFNN is then adopted here to estimate the $\Delta_{i}$ with $n$ inputs, one output and $N$ hidden neurons, expressed as $y=\boldsymbol{\theta}^{T} \boldsymbol{\xi}(\boldsymbol{x}) . \boldsymbol{x} \in \boldsymbol{R}^{N}$ is the input, $y$ is the output, $\boldsymbol{\theta} \in \boldsymbol{R}^{N}$ is the adjustable weight, and $\boldsymbol{\xi}(\boldsymbol{x}) \in \boldsymbol{R}^{N}$ is the nonlinear Gaussian basis function (GBF) which is expressed as $\boldsymbol{\xi}(\boldsymbol{x})=\left[\beta_{1}(\boldsymbol{x}), \ldots \beta_{N}\right]^{T}$, satisfying,

$$
\beta_{i}(x)=\exp \left(-\frac{\left\|\boldsymbol{x}-\boldsymbol{d}_{i}\right\|^{2}}{2 b^{2}}\right)
$$

where $\boldsymbol{d}_{i} \in \boldsymbol{R}^{N}$ and $b$ are the center and width of the $i^{t h}$ gaussian functions.

In this paper, the central coordinates and width of the GBF are pre-determined according to the error range of the UAV hardware design. The weight from the hidden layer to the output layer needs to be adjusted online to ensure that the RBF can track the error successfully. Assuming that $\Delta_{i}$ satisfies this equation,

$$
\Delta_{i}=\boldsymbol{\theta}_{i}{ }^{* T} \boldsymbol{\xi}_{i}\left(\mathbf{e}_{i}\right)+\sigma_{i}{ }^{*}
$$

where $\mathbf{e}_{i}=\left(x_{i 1}-x_{i d}, \dot{x}_{i 1}-\dot{x}_{i d}\right)^{T}, \boldsymbol{\xi}_{i}$ is the gaussian basis function, $\boldsymbol{\theta}_{i}{ }^{*}$ is the ideal weight and $\left\|\boldsymbol{\theta}_{i}{ }^{*}\right\| \leq \theta_{i M}, \sigma_{i}{ }^{*}$ is the approximation error and $\left|\sigma_{i}{ }^{*}\right| \leq \sigma_{i M}$. $\theta_{i M}$ and $\sigma_{i M}$ are both positive constants.

Then the RBF adaptive law is designed as,

$$
\dot{\hat{\boldsymbol{\theta}}}_{i}=\boldsymbol{\Gamma}_{i}\left[\boldsymbol{\xi}_{i}\left(\mathbf{e}_{i}\right) z_{i 2}-\eta_{i} \hat{\boldsymbol{\theta}}_{i}\right]
$$

where $\hat{\boldsymbol{\theta}}_{i}$ is the value to be updated, $\boldsymbol{\Gamma}_{i}$ is the constant matrix and $\eta_{i}$ is the design coefficient that affects the convergence rate of the neural network.

Then Eq.21) can be rewritten as,

$$
u_{i}=\frac{1}{b_{i}}\left(-f_{i}(x)+\dot{a}_{i}-\hat{\boldsymbol{\theta}}_{i}^{T} \boldsymbol{\xi}_{i}\left(\mathbf{e}_{i}\right)-k_{i 2} z_{i 2}-z_{i 1}\right)
$$


Input saturation is also a key factor that could affect the dynamic performance and even cause the UAV out of control [34. Therefore, the input saturation should be taken into account in the controller design. During the actual flight of the UAV, the actual control has upper and lower limits,

$$
u_{i m} \leq \bar{u}_{i} \leq u_{i n}
$$

where $\bar{u}_{i}$ is the actual control, $u_{i n}$ and $u_{i m}$ are the upper and lower bounds of $\bar{u}_{i}$.

When the controller output $u_{i}$ beyond the scope of the actual control $\bar{u}_{i}$, the anti-saturation auxiliary system is triggered to keep the output $u_{i}$ of the controller within the range of the actual control $\bar{u}_{i}$. The anti-saturation auxiliary system is designed as,

$$
\dot{\lambda}_{i}=\left\{\begin{array}{c}
-k_{i} \lambda_{i}-\frac{g_{i}(\cdot)}{\lambda_{i}}+\left(\bar{u}_{i}-u_{i}\right),\left|\lambda_{i}\right| \geq \varepsilon_{i} \\
0,\left|\lambda_{i}\right|<\varepsilon_{i}
\end{array}\right.
$$

where $g_{i}(\cdot)=g_{i}\left(z_{i 2}, \Delta u_{i}\right)=\left|z_{i 2} b_{i} \Delta u_{i}\right|+\frac{1}{2} \Delta u_{i}{ }^{2}, k_{i}>0, \Delta u_{i}=\bar{u}_{i}-u_{i}, \varepsilon_{i}$ is the minimal positive real number, $\lambda_{i}$ is the control correction parameter and $k_{i}$ is the designed parameter.

When $u_{i}$ does not exceed the range of $\bar{u}_{i}, \lambda_{i}$ is always 0 . If $u_{i}$ exceeds the range of $\bar{u}_{i}$, let the initial value of $\lambda_{i}$ be $\Delta u_{i} / b_{i}$, and item $g_{i}(\cdot) / \lambda_{i}$ will ensure that the output $u_{i}$ moves to the expected range. When $u_{i}$ falls back into the desired range, the item $-k_{i} \lambda_{i}$ will guarantee that $\lambda_{i}$ goes to 0 .

By adding the control correction $\lambda_{i}$ into Eq. 25), the final control law can be written as,

$$
u_{i}=\frac{1}{b_{i}}\left(-f_{i}(x)+\dot{a}_{i}-\hat{\boldsymbol{\theta}}_{i}^{T} \boldsymbol{\xi}_{i}\left(\mathbf{e}_{i}\right)-k_{i 2} z_{i 2}-z_{i 1}\right)+\lambda_{i}
$$


Based on Eqs.(8) (9) and (28), set $i=1,2,3,4,5,6$ respectively, the control law of the six controllers can be obtained accordingly.

\subsection{Stability analysis}

According to the principle of separability, it is proved that the stability of each subsystem can make the whole system in stable state. The first-order filter error is defined as,

$$
y_{i}=a_{i}-\bar{x}_{i 2}
$$

and the weight error is defined as,

$$
\tilde{\boldsymbol{\theta}}_{i}=\hat{\boldsymbol{\theta}}_{i}-\boldsymbol{\theta}_{i}{ }^{*}
$$

Define the Lyapunov function as,

$$
\left\{\begin{array}{c}
V_{i 2}=\frac{1}{2} y_{i}{ }^{2} \\
V_{i 3}=\frac{1}{2} \tilde{\boldsymbol{\theta}}_{i}^{T} \boldsymbol{\Gamma}_{i}^{-1} \tilde{\boldsymbol{\theta}}_{i} \\
V_{i 4}=\frac{1}{2} \lambda_{i}{ }^{2} \\
V_{i}=V_{i 1}+V_{i 2}+V_{i 3}+V_{i 4} \\
=\frac{1}{2}\left(z_{i 1}^{2}+z_{i 2}^{2}\right)+\frac{1}{2} y_{i}{ }^{2}+\frac{1}{2} \tilde{\boldsymbol{\theta}}_{i}^{T} \boldsymbol{\Gamma}_{i}^{-1} \tilde{\boldsymbol{\theta}}_{i}+\frac{1}{2} \lambda_{i}{ }^{2}
\end{array}\right.
$$

Then the derivation of $V_{i 1}$ is written as,

$$
\begin{aligned}
\dot{V}_{i 1} & =z_{i 1} \dot{z}_{i 1}+z_{i 2} \dot{z}_{i 2}=z_{i 1}\left(z_{i 2}+y_{i}+\bar{x}_{i 2}-\dot{x}_{i d}\right) \\
& +z_{i 2}\left(f_{i}(x)+b_{i} u_{i}+\Delta_{i}-\dot{a}_{i}\right) \\
& =z_{i 1}\left(z_{i 2}+y_{i}-k_{i 1} z_{i 1}\right) \\
& +z_{i 2}\left(b_{i} \Delta u_{i}+\lambda_{i}-k_{i 2} z_{i 2}-z_{i 1}+\Delta_{i}-\hat{\boldsymbol{\theta}}_{i}^{\mathbf{T}} \boldsymbol{\xi}_{i}\left(\mathbf{e}_{i}\right)\right) \\
& =z_{i 1} y_{i}-k_{i 1} z_{i 1}^{2}+z_{i 2} b_{i} \Delta u_{i} \\
& +z_{i 2} \lambda_{i}-k_{i 2} z_{i 2}^{2}-z_{i 2} \tilde{\boldsymbol{\theta}}_{i}^{\mathbf{T}} \boldsymbol{\xi}_{i}\left(\mathbf{e}_{i}\right)+z_{i 2} \sigma_{i}{ }^{*}
\end{aligned}
$$


The derivation of $y_{i}$ can be calculated as,

$$
\dot{y}_{i}=\dot{a}_{i}-\dot{\bar{x}}_{i 2}=-\frac{y_{i}}{\tau_{i}}+k_{i 1} \dot{z}_{i 1}-\ddot{x}_{i d}
$$

According to Eq. (33), there is a continuous nonnegative continuous function $B_{i 2}$ and constant $M_{i 2}$,

$$
\left|\dot{y}_{i}+\frac{y_{i}}{\tau_{i}}\right| \leq B_{i 2}\left(\dot{z}_{i 1}, \ddot{x}_{i d}\right) \leq M_{i 2}
$$

Then,

$$
\dot{V}_{i 2}=y_{i} \dot{y}_{i} \leq-\frac{y_{i}^{2}}{\tau_{i}}+B_{i 2}\left|y_{i}\right|
$$

The derivation of $V_{i 3}$ is calculated as,

$$
\begin{aligned}
\dot{V}_{i 3} & =\tilde{\boldsymbol{\theta}}_{i}^{T} \boldsymbol{\Gamma}_{i}^{-1} \dot{\tilde{\boldsymbol{\theta}}}_{i} \\
& =\tilde{\boldsymbol{\theta}}_{i}^{T} \boldsymbol{\Gamma}_{i}^{-1}\left(\boldsymbol{\Gamma}_{i}\left[\boldsymbol{\xi}_{i}(\mathbf{e}) z_{i 2}-\eta_{i} \hat{\boldsymbol{\theta}}_{i}\right]\right) \\
& =z_{i 2} \tilde{\boldsymbol{\theta}}_{i}^{T} \boldsymbol{\xi}_{i}(\mathbf{e})-\eta_{i} \tilde{\boldsymbol{\theta}}_{i}^{T} \hat{\boldsymbol{\theta}}_{i}
\end{aligned}
$$

and the derivation of $V_{i 4}$ is calculated as,

$$
\dot{V}_{i 4}=\lambda_{i} \dot{\lambda}_{i} \leq-k_{i} \lambda_{i}{ }^{2}-\frac{\left|z_{i 2} b_{i} \Delta u_{i}\right|+\frac{1}{2} \Delta u_{i}{ }^{2}}{\lambda_{i}{ }^{2}} \lambda_{i}{ }^{2}+\Delta u_{i} \lambda_{i}
$$

Based on the existence inequality principle, it has,

$$
\Delta u_{i} \cdot \lambda_{i} \leq \frac{1}{2} \Delta u_{i}^{2}+\frac{1}{2} \lambda_{i}^{2}
$$

Substituting Eq.(38) into Eq.(37), it can obtain,

$$
\dot{V}_{i 4} \leqslant-\left(k_{i}-\frac{1}{2}\right) \lambda_{i}{ }^{2}-z_{i 2} b_{i} \Delta u_{i}
$$


At the same time, the weights of the neural network follow the equation,

$$
2 \tilde{\boldsymbol{\theta}}_{i}^{T} \hat{\boldsymbol{\theta}}_{i} \geq\left\|\tilde{\boldsymbol{\theta}}_{i}^{T}\right\|^{2}-\left\|\boldsymbol{\theta}_{i}^{*}\right\|^{2}
$$

According to Eqs. 32) (35) (36) (39) (40),

$$
\begin{aligned}
\dot{V}_{i} & \leq z_{i 1} y_{i}-k_{i 1} z_{i 1}^{2}+z_{i 2} \lambda_{i}-k_{i 2} z_{i 2}^{2}+z_{i 2} \sigma_{i}{ }^{*}-\frac{y_{i}{ }^{2}}{\tau_{i}} \\
& +B_{i 2}\left|y_{i}\right|-\eta_{i} \tilde{\boldsymbol{\theta}}_{i}^{\mathbf{T}} \hat{\boldsymbol{\theta}}_{i}-\left(k_{i}-\frac{1}{2}\right) \lambda_{i}{ }^{2} \\
& \leq \frac{1}{2} z_{i 1}^{2}+\frac{1}{2} y_{i}{ }^{2}-k_{i 1} z_{i 1}^{2}+\frac{1}{2} z_{i 2}^{2}+\frac{1}{2} \lambda_{i}{ }^{2}-k_{i 2} z_{i 2}^{2}+\frac{1}{2} z_{i 2}^{2} \\
& +\frac{1}{2} \sigma_{i}{ }^{* 2}-\frac{y_{i}{ }^{2}}{\tau_{i}}+\frac{B_{i 2}^{2} y_{i}{ }^{2}}{2 \varepsilon_{i}}+\frac{\varepsilon_{i}}{2} \\
& -\frac{\eta_{i}}{2}\left(\left\|\tilde{\boldsymbol{\theta}}_{i}^{T}\right\|^{2}-\left\|\boldsymbol{\theta}_{i}{ }^{*}\right\|^{2}\right)-\left(k_{i}-\frac{1}{2}\right) \lambda_{i}{ }^{2} \\
& \leq\left(\frac{1}{2}-k_{i 1}\right) z_{i 1}^{2}+\left(1-k_{i 2}\right) z_{i 2}^{2}+\left(\frac{1}{2}-\frac{1}{\tau_{i}}+\frac{B_{i 2}^{2}}{2 \varepsilon_{i}}\right) y_{i}{ }^{2} \\
& -\frac{\eta_{i}}{2 \mu_{i \max }\left(\Gamma^{-1}\right)} \tilde{\boldsymbol{\theta}}_{i}^{T} \boldsymbol{\Gamma}_{i}^{-1} \tilde{\boldsymbol{\theta}}_{i}-\left(k_{i}-\frac{1}{2}\right) \lambda_{i}{ }^{2}+\frac{1}{2} \sigma_{i}{ }^{* 2} \\
& +\frac{\varepsilon_{i}}{2}+\frac{\eta_{i}}{2}\left\|\boldsymbol{\theta}_{i}{ }^{*}\right\|^{2}
\end{aligned}
$$

where $2 \mu_{i \max }\left(\boldsymbol{\Gamma}_{i}^{-1}\right)$ is the maximum eigenvalue of $\boldsymbol{\Gamma}_{i}{ }^{-\mathbf{1}}$. The ranges of the involved control parameters are limited as,

$$
\left\{\begin{array}{c}
k_{i 1}>\frac{1}{2}+r_{i} \\
k_{i 2}>1+r_{i} \\
\frac{1}{\tau_{i}}>\frac{1}{2}+\frac{M_{i 2}^{2}}{2 \varepsilon_{i}}+r_{i} \\
k_{i}>r_{i}+\frac{1}{2} \\
\eta_{i}>2 r_{i} \mu_{i \max }\left(\boldsymbol{\Gamma}_{i}^{-1}\right)
\end{array}\right.
$$


Table 1: UAV model parameters

\begin{tabular}{ccc|ccc}
\hline Para & Value & Unit & Para & Value & Unit \\
\hline$m$ & 0.650 & $\mathrm{~kg}$ & $I_{y}$ & $7.5 \mathrm{e}-3$ & $\mathrm{~kg} \cdot \mathrm{m}^{2}$ \\
$l$ & 0.23 & $m$ & $I_{z}$ & $1.3 \mathrm{e}-2$ & $\mathrm{~kg} \cdot \mathrm{m}^{2}$ \\
$g$ & 9.81 & $\mathrm{~m} / \mathrm{s}^{2}$ & $b$ & $3.13 \mathrm{e}-5$ & $\mathrm{~N} \cdot \mathrm{s}^{2}$ \\
$I_{x}$ & $7.5 \mathrm{e}-3$ & $\mathrm{~kg} \cdot \mathrm{m}^{2}$ & $d$ & $7.5 \mathrm{e}-7$ & $\mathrm{~N} \cdot \mathrm{ms}^{2}$ \\
\hline
\end{tabular}

where $r_{i}$ is a positive constant. Hence, Eq.41 can be written as follows,

$$
\begin{aligned}
\dot{V}_{i} & \leq-r_{i} z_{i 1}^{2}-r_{i} z_{i 2}^{2}-r_{i} y_{i}{ }^{2}-r_{i} \tilde{\boldsymbol{\theta}}_{i}^{T} \boldsymbol{\Gamma}_{i}{ }^{-1} \tilde{\boldsymbol{\theta}}_{i}+Q_{i} \\
& =-2 r_{i} V_{i}+Q_{i}
\end{aligned}
$$

where $Q_{i}=\frac{1}{2} \sigma_{i M}^{2}+\frac{\varepsilon_{i}}{2}+\frac{\eta_{i}}{2} \theta_{i M}^{2}$. Eq.43 can be further transformed into the form as,

$$
V_{i} \leq \frac{Q_{i}}{2 r_{i}}+\left(V_{i}(0)-\frac{Q_{i}}{2 r_{i}}\right) e^{-2 r_{i} t}
$$

According to the Lyapunov stability theory and Eq. 44, by setting reasonable controller parameters, the semi-global asymptotic stability of the whole closed-loop system can be guaranteed, and the system's trajectory tracking error and state are bounded accordingly.

\section{Experimental results and analysis}

To verify the efficacy of the proposed control algorithm, trajectory tracking, anti-disturbance and anti-saturation simulation experiments are carried out by MATLAB software. The parameters of the UAV model used in this paper are listed in Table I.

The UAV rotor speed used in the simulation experiments has an upper limit of 955 RPM. According to Eq. (4), the following constraints of the control force 
are set as,

$$
\left\{\begin{aligned}
0 & \leq U_{1} \leq 107 \\
-6 & \leq U_{2} \leq 6 \\
-6 & \leq U_{3} \leq 6 \\
-1.28 & \leq U_{4} \leq 1.28
\end{aligned}\right.
$$

To ensure the safety and stability of the UAV flight, the desired roll $\varphi_{d}$ and pitch $\theta_{d}$ should be limited within the range $\left[-30^{\circ}, 30^{\circ}\right]$. Based on the knowledge of trigonometric functions, $U_{x}$ and $U_{y}$ can be rewritten as,

$$
\begin{aligned}
& U_{x}=\left(A^{2}+B^{2}\right)^{\frac{1}{2}} \sin \left(\psi+t_{1}\right), \sin \phi=A, \sin \theta \cos \phi=B \\
& U_{y}=\left(B^{2}+C^{2}\right)^{\frac{1}{2}} \sin \left(\psi+t_{2}\right),-\sin \phi=C, \tan t_{2}=\frac{C}{B}
\end{aligned}
$$

where $\tan t_{1}=\frac{B}{A}$. Since $\sin (\psi+t)$ ranges from -1 to $1, U_{x}$ and $U_{y}$ have the following inequality constraints,

$$
\begin{aligned}
& -\left(\sin ^{2} \theta \cos ^{2} \phi+\sin ^{2} \phi\right)^{\frac{1}{2}} \leq U_{x} \leq\left(\sin ^{2} \theta \cos ^{2} \phi+\sin ^{2} \phi\right)^{\frac{1}{2}} \\
& -\left(\sin ^{2} \theta \cos ^{2} \phi+\sin ^{2} \phi\right)^{\frac{1}{2}} \leq U_{y} \leq\left(\sin ^{2} \theta \cos ^{2} \phi+\sin ^{2} \phi\right)^{\frac{1}{2}}
\end{aligned}
$$

According to Eq. 47] and the constraints of the desired roll $\varphi_{d}$ and pitch $\theta_{d}$, $U_{x}$ and $U_{y}$ are limited as,

$$
\left\{\begin{array}{l}
-1.32 \leq U_{x} \leq 1.32 \\
-1.32 \leq U_{y} \leq 1.32
\end{array}\right.
$$

Considering the control parameter constraints of Eq. 42 , in the actual simulation experiments, the selected control parameter values are listed in Table2.

\subsection{The experiments of trajectory tracking}

In order to prove the high accuracy of the trajectory tracking capability of the proposed control algorithm, other typical control methods are used for 
Table 2: Control parameters of the UAV

\begin{tabular}{c|ccccc}
\hline & $k_{1}$ & $k_{2}$ & $\tau$ & $k$ & $\eta$ \\
\hline$x$ & 1.2 & 4 & 0.02 & 80 & 0.02 \\
$y$ & 1.2 & 4 & 0.02 & 80 & 0.02 \\
$z$ & 1.2 & 4 & 0.02 & 80 & 0.04 \\
$\varphi$ & 10 & 10 & 0.02 & 100 & 0.4 \\
$\theta$ & 10 & 10 & 0.02 & 100 & 0.4 \\
$\psi$ & 10 & 10 & 0.02 & 100 & 0.4 \\
\hline
\end{tabular}

comparison. Spiraling is a difficult trajectory for UAV to track, so the reference trajectories adopted in the experiments are selected as follows,

$$
\left\{\begin{array}{c}
x_{d}=0.5 \sin (0.5 t+0.5) \\
y_{d}=0.5 \sin (0.5 t) \\
z_{d}=2+0.1 t \\
\psi_{d}=\pi / 6
\end{array}\right.
$$
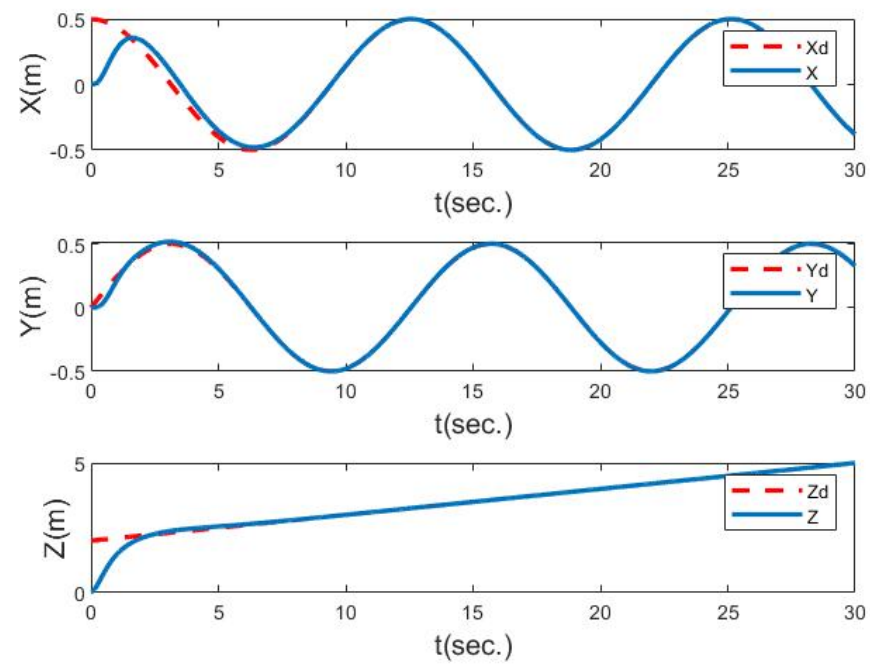

Figure 3: Position tracking under the proposed control algorithm 

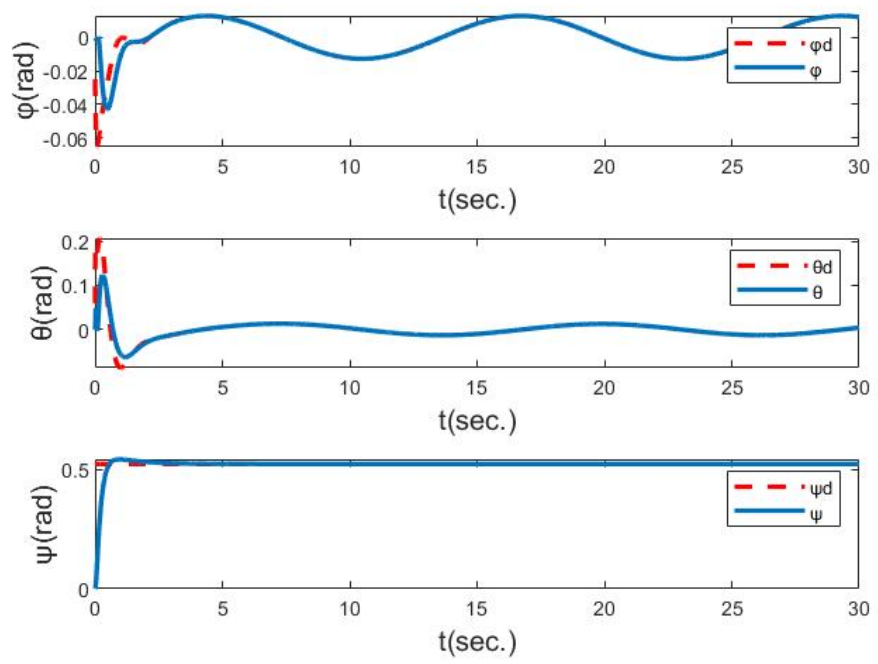

Figure 4: Position tracking under the proposed control algorithm

Fig. 3 and Fig. 4 demonstrate the position and attitude tracking of the UAV under the proposed control algorithms. It can be seen from Fig. 3 and Fig. 4 that the actual attitude can track the expected attitude with a small error after flying $2 \mathrm{~s}$. After about $6 \mathrm{~s}$ of the flight, there is no obvious error between the actual and the expected position.

PD, LQR and backstepping control methods are used for performance comparison, while the integral of the absolute error (IAE) is used as the criterion to evaluate the error of the trajectory tracking control capability. The IAE values of different degree of freedom (DoF) of the compared control methods are shown in Table 3 ,

It should be noted that PD and LQR are linear control methods, while backstepping and the proposed controller are nonlinear control methods. According to Table 3 the IAE values of the nonlinear control methods is much lower than those of the linear control methods. Moreover, the IAE value of the proposed control method is similar to that of the backstepping method, which indicates 
Table 3: IAE values of each DoF

\begin{tabular}{lllll}
\hline DoF & PD & LQR & Backstepping & $\begin{array}{l}\text { Proposed } \\
\text { method }\end{array}$ \\
\hline$x$ & 1.673 & 5.209 & 0.650 & 0.798 \\
$y$ & 1.03 & 4.526 & 0.237 & 0.166 \\
$z$ & 2.502 & 5.933 & 2.019 & 1.908 \\
$\varphi$ & 0.061 & 0.057 & 0.018 & 0.027 \\
$\theta$ & 0.157 & 0.105 & 0.018 & 0.053 \\
$\psi$ & 0.549 & 0.155 & 0.090 & 0.130 \\
\hline
\end{tabular}

that the introduction of first-order low-pass filter does not affect the tracking performance of the UAV.

By comparison analysis, it is proved that the proposed control method can follow the expected trajectory with small error and the trajectory tracking capability is better than those of PD and LQR linear control methods.

\subsection{The experiments of anti-disturbance control}

In this paper, the designed control algorithm uses the RBF neural network to estimate and compensate the disturbance, so as to achieve satisfied disturbance rejection.

Thus, based on the reference trajectory of Eq. 49, the following disturbance is added in the experiments in order to illustrate the effectiveness of the RBF neural network.

$$
\left\{\begin{array}{l}
\Delta_{1}=0.3+0.1 \sin t \\
\Delta_{2}=0.5+0.1 \sin t \\
\Delta_{3}=1.0+0.1 \sin t \\
\Delta_{4}=0.5+0.1 \sin t \\
\Delta_{5}=0.5+0.1 \sin t \\
\Delta_{6}=0.5+0.1 \sin t
\end{array}\right.
$$



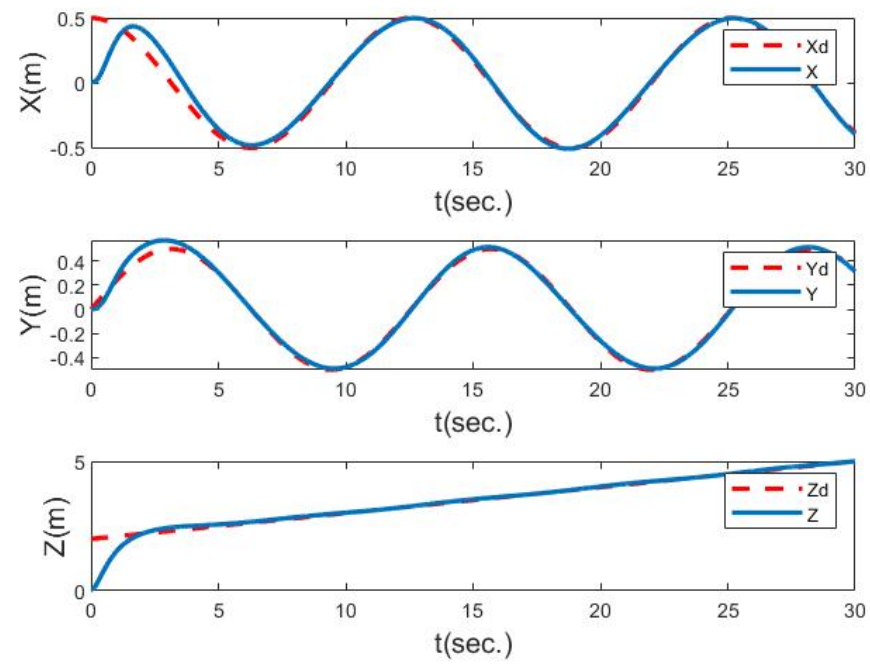

Figure 5: Position tracking with the RBF neural network
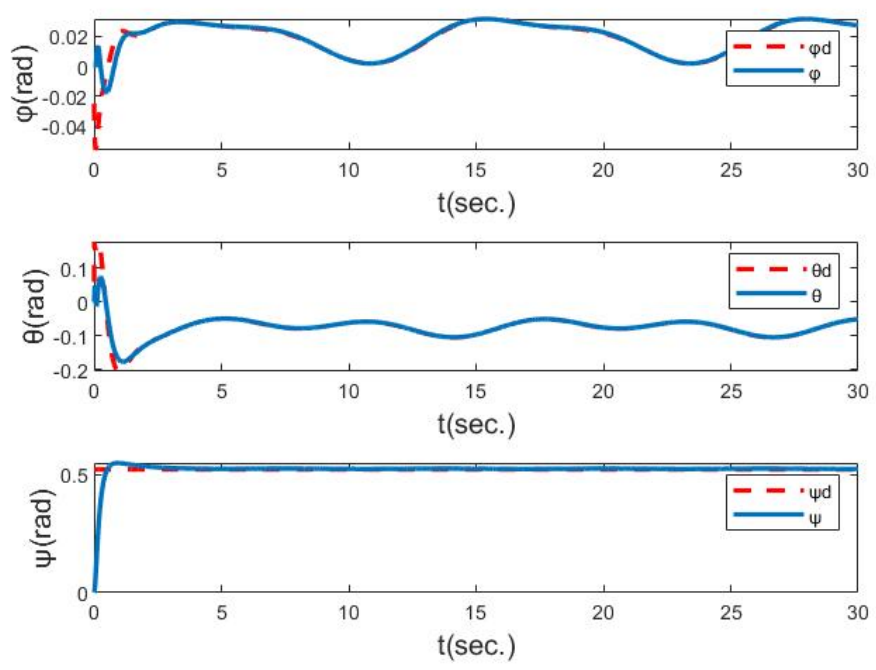

Figure 6: Attitude tracking with the RBF neural network 

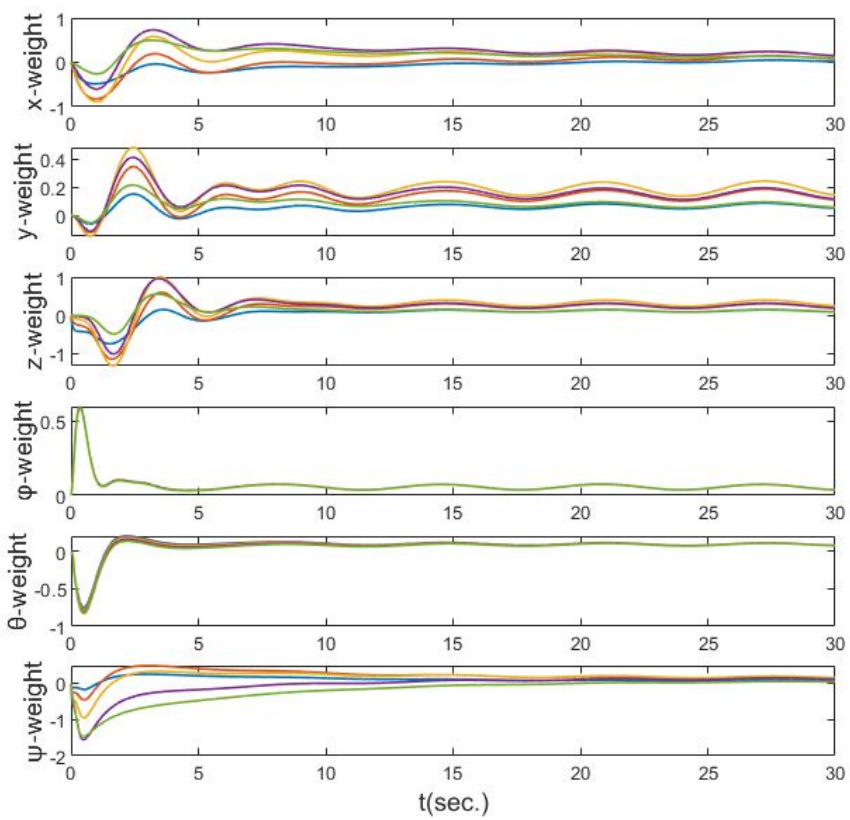

Figure 7: RBF neural network weight adjustment 


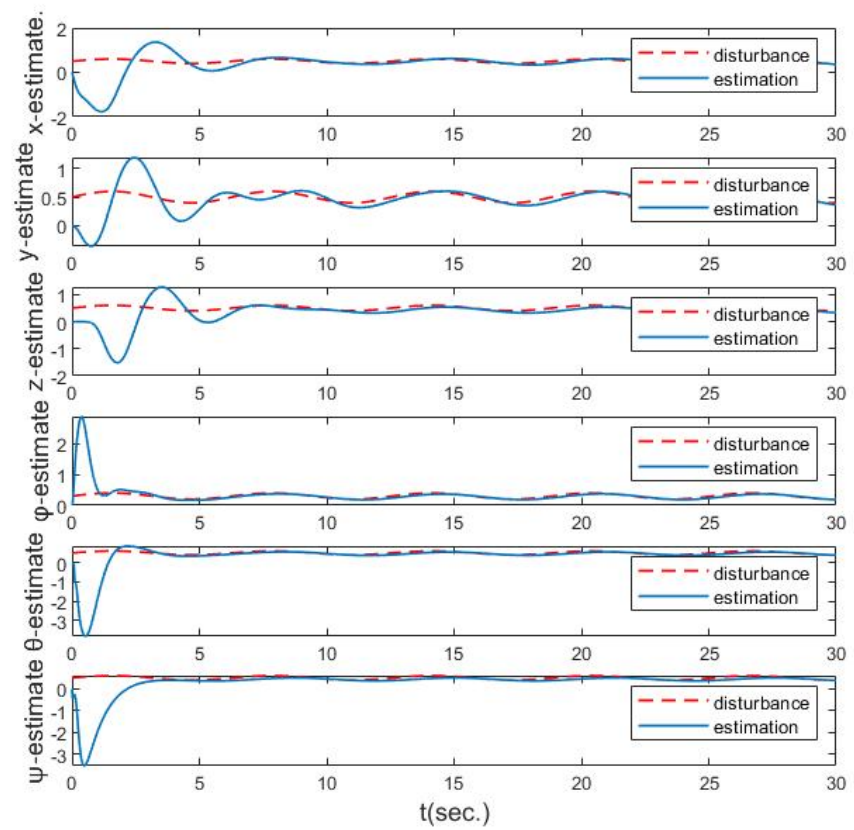

Figure 8: RBF neural network estimation

The position and attitude tracking of the UAV with the RBF neural network are shown in Fig. 5 and Fig. 6, which can demonstrate that the actual and the desired trajectories of each DoF basically can coincide after $6 \mathrm{~s}$. The results of the weight adjustment and error estimation with RBF neural network are demonstrated in Fig. 7 and Fig. 8. As seen in Fig. 8, the RBF neural network can estimate the actual disturbance with a relatively small error after a period of adjustment. Combined with Fig. 7] it can be concluded that RBF neural network does play an important role in the disturbance observer.

Similarly, to evaluate the performance of the trajectory tracking control more accurately, the IAE values of each DoF of different control methods is calculated, which are demonstrated in Table 4.

According to the data in Table 3 and Table 4 , the tracking capability of the 


\begin{tabular}{llclll}
\multicolumn{7}{c}{ Table 4: IAE value of each DoF } \\
\hline DoF & PD & LQR & $\begin{array}{l}\text { Backstep } \\
\text {-ping }\end{array}$ & $\begin{array}{l}\text { Proposed } \\
\text { method } \\
\text { without } \\
\text { RBF }\end{array}$ & $\begin{array}{l}\text { Proposed } \\
\text { method } \\
\text { with } \\
\text { RBF }\end{array}$ \\
\hline$x$ & 11.540 & 8.554 & 5.548 & 3.071 & 1.119 \\
$y$ & 7.675 & 5.531 & 4.350 & 2.501 & 0.541 \\
$z$ & 8.567 & 3.141 & 6.128 & 3.880 & 2.217 \\
$\varphi$ & 0.425 & 0.310 & 0.101 & 0.108 & 0.032 \\
$\theta$ & 0.696 & 0.550 & 0.156 & 0.194 & 0.080 \\
$\psi$ & 1.631 & 1.129 & 0.382 & 0.394 & 0.204 \\
\hline
\end{tabular}

four control methods without disturbance observer would be seriously reduced after disturbance injection. At the same time, the IAE of the proposed control method with RBF is much smaller than that of proposed method without $\mathrm{RBF}$ and other control methods. Therefore, the experimental results can verify that the RBF neural network can estimate the disturbance effectively and the proposed control method has good disturbance rejection capability.

\subsection{The anti-saturation control experiments}

Under certain extreme conditions, the controller output may exceed the actual control range which is negative for the trajectory tracking control. In order to verify the effectivess of the proposed anti-saturation input strategy, the $z$ DoF is taken as an example for the experiments. The reference trajectory is set as follows,

$$
\left\{\begin{array}{c}
x_{d}=0.5 \sin (0.5 t+0.5) \\
y_{d}=0.5 \sin (0.5 t) \\
z_{d}=9+0.1 t \\
\psi_{d}=\pi / 6
\end{array}\right.
$$




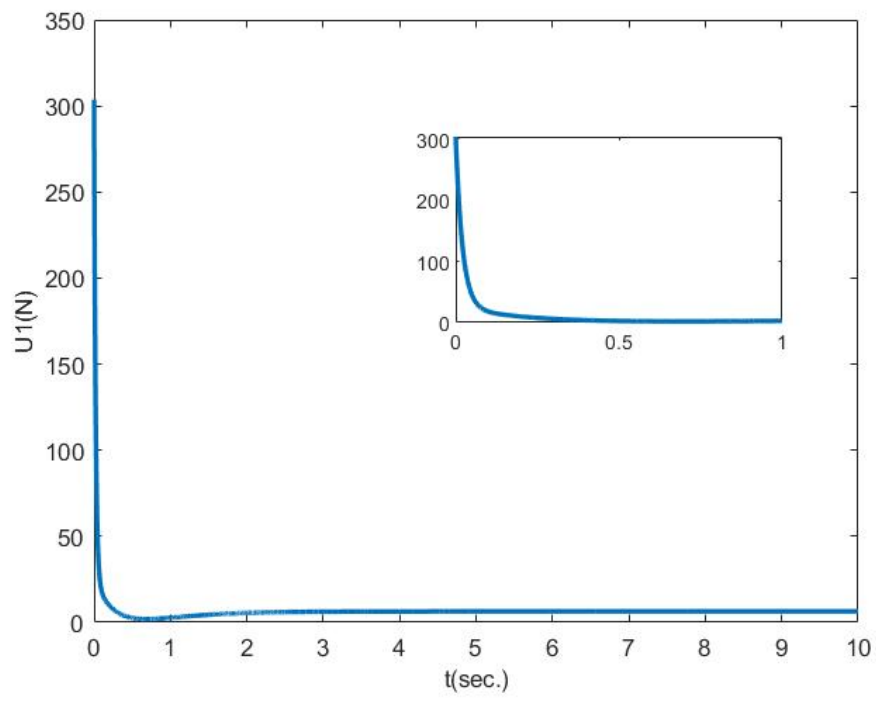

Figure 9: The $U_{1}$ output without anti-saturation module

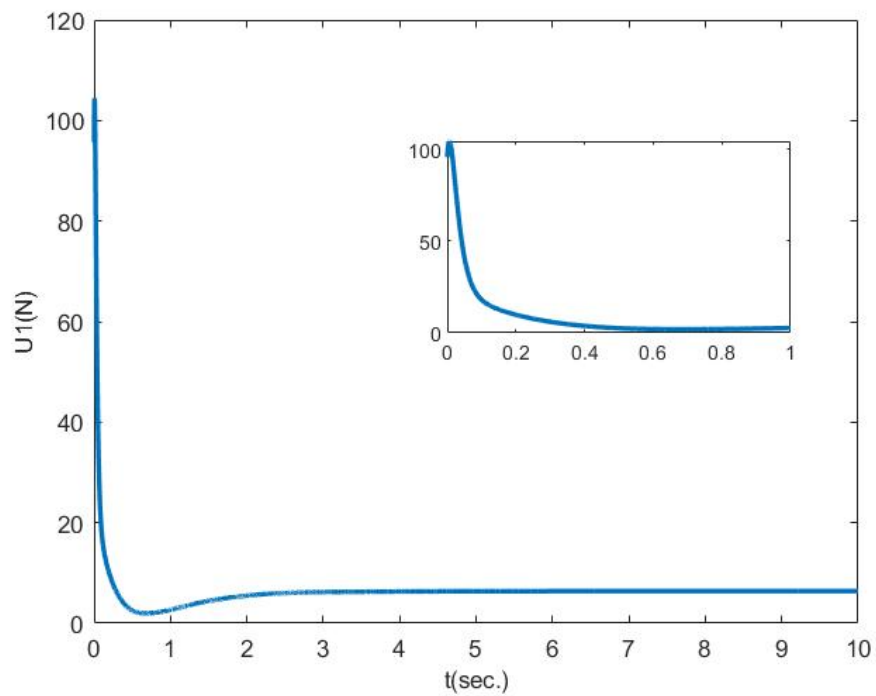

Figure 10: The $U_{1}$ output with anti-saturation module 


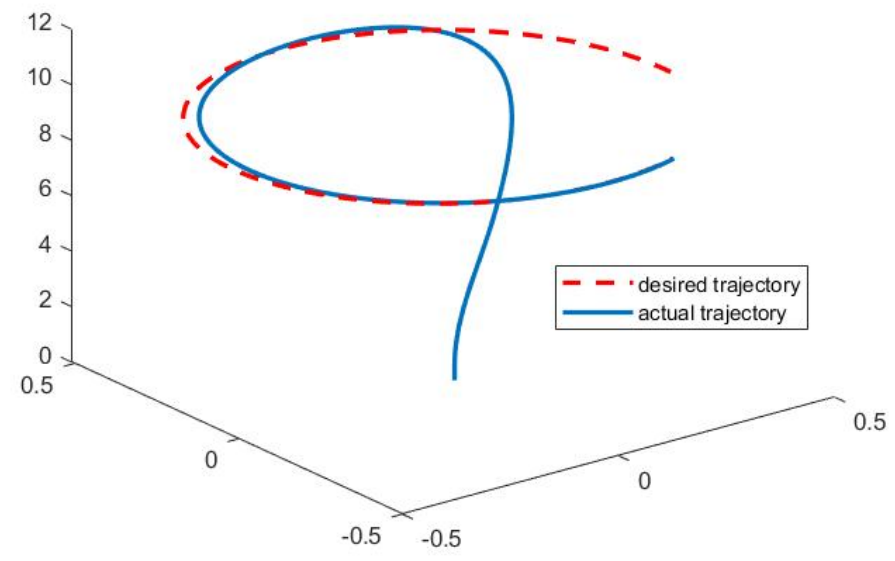

Figure 11: The desired trajectory and actual trajectory with anti-saturation module

The controller output $U_{1}$ of the altitude subsystem $z$ without anti-saturation module is shown in Fig. 9 Fig. 10 and Fig. 11 represent the controller output $U_{1}$ of the altitude subsystem $z$ with anti-saturation module and 3D trajectory tracking respectively. According to Eq.45, the upper limit of $U_{1}$ is 107. However, it can be seen from Fig. 9 that the controller output $U_{1}$ exceeds 107 at the initial stage. After adding the anti-saturation module, it is shown from Fig. 10 that $U_{1}$ is limited within the range of 107. Meanwhile, Fig. 11 displays that the UAV can still track steadily after the addition of the anti-saturation module.

Therefore, the addition of the anti-saturation module will not damage the stability of UAV, and can solve the problem of input saturation effectively.

\section{Conclusion}

In this paper, an adaptive dynamic surface control method based on RBF neural network and anti-saturation input system is proposed to solve the prob- 
lem of UAV trajectory tracking control under unknown time-varying interference and input saturation limitation during the flight. The dynamic surface control method is adopted so as to ensure the UAV track tracking capability. Then the universal approximation ability of RBF neural network is applied to estimate and compensate the total disturbance and improve the anti-disturbance ability of the system. Anti-saturation module is also introduced to solve the problem of UAV input saturation. The experimental results demonstrate that the designed system has good dynamic performance, strong anti-disturbance performance and reasonable control force.

Further study will investigate the parameter optimization of the developed control algorithm wtih actual UAV flight control in more complex field experiments.

\section{Acknowledgement}

This study was funded by the National Natural Science Foundation of China (NSFC) (grant number 61973296 and U1913201) and Shenzhen Basic Research Program (grant number JCYJ20170818153635759).

\section{Data Availability Statements}

Data sharing not applicable to this article as no datasets were generated or analysed during the current study.

\section{Conflict of Interest}

The authors declare that they have no conflict of interest. 


\section{References}

[1] J. Kim, S. A. Gadsden and S. A. Wilkerson, A Comprehensive Survey of Control Strategies for Autonomous Quadrotors, Canadian Journal of Electrical and Computer Engineering. 43 (2020) 3-16.

[2] H. Shraim, A. Awada and R. Youness, A survey on quadrotors: Configurations, modeling and identification, control, collision avoidance, fault diagnosis and tolerant control, IEEE Aerospace and Electronic Systems Magazine. 33 (2018) 14-33.

[3] A. Benaddy, M. Bouzi and M. Labbadi, Comparison of the different control strategies for Quadrotor Unmanned Aerial Vehicle, 2020 International Conference on Intelligent Systems and Computer Vision. (2020) 1-6.

[4] F. Favarim, R. Cris Brito, K. S. C. Linares and E. Todt, Comparison Analysis between PID and Fuzzy Logic Controllers for Quadrotor in a Simulated and in a Real Environment, 2018 Latin American Robotic Symposium, 2018 Brazilian Symposium on Robotics (SBR) and 2018 Workshop on Robotics in Education. (2018) 490-495.

[5] M. Nguyen Duc, T. N. Trong and Y. S. Xuan, The quadrotor MAV system using PID control, 2015 IEEE International Conference on Mechatronics and Automation. (2015) 506-510.

[6] Mahmoodabadi, M. and N. R. Babak, Robust fuzzy linear quadratic regulator control optimized by multi-objective high exploration particle swarm optimization for a 4 degree-of-freedom quadrotor, Aerospace Science and Technology. 97 (2020) 105598.

[7] O. J. Gonzalez Villarreal, J. A. Rossiter and H. Shin, Laguerre-Based Adap- 
tive MPC for Attitude Stabilization of Quad-Rotor, 2018 UKACC 12th International Conference on Control. (2018) 360-365.

[8] M. Emam and A. Fakharian, Attitude tracking of quadrotor UAV via mixed $H_{2} / H_{\infty}$ controller: An LMI based approach, 2016 24th Mediterranean Conference on Control and Automation. (2016) 390-395.

[9] J. Gao, Y. Zhuang, J. Xiao and Y. Zhao, Attitude tracking control of a quadrotor based on linear active disturbance rejective control, 2015 IEEE International Conference on Cyber Technology in Automation, Control, and Intelligent Systems. (2015) 287-292.

[10] C. Rosales, S. Tosetti, C. Soria and F. Rossomando, Neural Adaptive PID Control of a Quadrotor using EFK, IEEE Latin America Transactions. 16 (2018) 2722-2730.

[11] O. García, P. Ordaz, O. Santos-Sánchez, S. Salazar and R. Lozano, Backstepping and Robust Control for a Quadrotor in Outdoors Environments: An Experimental Approach, IEEE Access. 7 (2019) 40636-40648.

[12] Q. Jing, Z. Chang, H. Chu, Y. Shao and X. Zhang, Quadrotor Attitude Control Based on Fuzzy Sliding Mode Control Theory, 2019 Chinese Control Conference. (2019) 8360-8364.

[13] H. Wang, X. Ye, Y. Tian, G. Zheng and N. Christov, Model-freebased terminal SMC of quadrotor attitude and position, IEEE Transactions on Aerospace and Electronic Systems. 52 (2016) 2519-2528.

[14] Y. Wang, H. Zhang and D. Han, Neural network adaptive inverse model control method for quadrotor UAV, 2016 35th Chinese Control Conference. (2016) 3653-3658. 
[15] Xingling, Shao et al. Estimator-based MLP neuroadaptive dynamic surface containment control with prescribed performance for multiple quadrotors, Aerospace Science and Technology. 97 (2020) 105620.

[16] A. Zolotas, Disturbance Observer-Based Control: Methods and Applications [Bookshelf], IEEE Control Systems. 35 (2015) 55-57.

[17] N. Ahmed, M. Chen and S. Shao, Disturbance Observer Based Tracking Control of Quadrotor With High-Order Disturbances, IEEE Access. 8 (2020) 8300-8313.

[18] L. Qian and H. H. T. Liu, Path-Following Control of A Quadrotor UAV With A Cable-Suspended Payload Under Wind Disturbances, IEEE Transactions on Industrial Electronics. 67 (2020) 2021-2029.

[19] B. Xiao and S. Yin, A New Disturbance Attenuation Control Scheme for Quadrotor Unmanned Aerial Vehicles, IEEE Transactions on Industrial Informatics. 13 (2017) 2922-2932.

[20] S. Abiko and K. Tashiro, Fundamental numerical and experimental evaluation of attitude recovery control for a quad tilt rotor UAV against disturbance, 2016 16th International Conference on Control, Automation and Systems. (2016) 709-712.

[21] S. Shao, M. Chen and Y. Zhang, Adaptive Discrete-Time Flight Control Using Disturbance Observer and Neural Networks, IEEE Transactions on Neural Networks and Learning Systems. 30 (2019) 3708-3721.

[22] D. Cabecinhas, R. Cunha and C. Silvestre, A Globally Stabilizing Path Following Controller for Rotorcraft With Wind Disturbance Rejection, IEEE Transactions on Control Systems Technology. 23 (2015) 708-714. 
[23] Huang Y, Zheng Z, Sun L, et al, Saturated adaptive sliding mode control for autonomous vessel landing of a quadrotor, Iet Control Theory and Applications. 12 (2018) 1830-1842.

[24] Bernstein DS, MichelAN, Achronological bibliography on saturating actuators, International Journal of Robust and Nonlinear Control. 5 (2020) $375-80$.

[25] S. Herdjunanto, G. N. P. Pratama, A. P. Sandiwan and A. I. Cahyadi, Quadrotor Proportional-Derivative Control on $\mathrm{SO}(3)$ with Input Saturation, 2018 12th South East Asian Technical University Consortium. 1 (2018) $1-5$.

[26] H. Liu, B. Lyu, H. Zheng and C. Yang, Suboptimal Integral Sliding Mode Control of a Quadrotor under Input Saturation, 2018 IEEE CSAA Guidance, Navigation and Control Conference. (2018) 1-7.

[27] Z. Zhao, Z. Zheng, M. Zhu and Z. Wu, Adaptive fault tolerant attitude tracking control for a quadrotor with input saturation and full-state constraints, 2017 13th IEEE International Conference on Control \& Automation. (2017) 46-51.

[28] M. Faessler, D. Falanga and D. Scaramuzza, Thrust Mixing, Saturation, and Body-Rate Control for Accurate Aggressive Quadrotor Flight, IEEE Robotics and Automation Letters. 2 (2017) 476-482.

[29] Wang, J. et al. Adaptive event-triggered control for quadrotor aircraft with output constraints, Aerospace Science and Technology. 105 (2020) 105935.

[30] Su, Yuxin, and Jan Swevers. Finite-time tracking control for robot manipulators with actuator saturation, Robotics and Computer-integrated Manufacturing. 30 (2012) 91-98. 
[31] H. Ma, H. Liang, Q. Zhou and C. K. Ahn, Adaptive Dynamic Surface Control Design for Uncertain Nonlinear Strict-Feedback Systems With Unknown Control Direction and Disturbances, IEEE Transactions on Systems, Man, and Cybernetics: Systems. 49 (2019) 506-515.

[32] D. Swaroop, J. K. Hedrick, P. P. Yip and J. C. Gerdes, Dynamic surface control for a class of nonlinear systems, IEEE Trans. Autom. Control. 45 (2000) 1893-1899.

[33] J. Park and I. W. Sandberg, Universal approximation using radial-basisfunction networks, Neural Computation. 3 (1991) 246-257.

[34] Y. Gao, X. Sun, C. Wen and W. Wang, Adaptive Tracking Control for a Class of Stochastic Uncertain Nonlinear Systems With Input Saturation, IEEE Transactions on Automatic Control. 62 (2017) 2498-2504. 